\title{
Some Novel Ways for Neutrino Mass Generation
}

\author{
Nick E. Mavromatos ${ }^{* \dagger}$ \\ King's College London, Physics Department, Theoretical Particle Physics and Cosmology \\ Group, Strand, London WC2R 2LS, UK \\ E-mail: Nikolaos.Mavromatos@cern.ch
}

\begin{abstract}
I discuss dynamical generation of neutrino masses in unconventional scenarios where the background space-time geometry plays a crucial role. I discuss two types of backgrounds: (i) Lorentz Violating and (ii) Geometries with Torsion. In the former case, the violation of Lorentz symmetry, at a scale M, may be viewed as a catalyst for mass generation and induced flavour oscillations among neutrino species, which survive the limit of $M$ taken to infinity, leading to a hierarchy among neutrino masses. In the latter case, the (totally antisymmetric components of the) torsion degrees of freedom correspond to a pseudoscalar axion field in four space-time dimensions. This field is assumed to be mixed, through non-diagonal kinetic terms, with ordinary axion fields that may exist in the theory for other reasons and couple to neutrinos with chirality changing Yukawa couplings. The torsion-ordinary-axion-field mixing is responsible, through higher-loop anomalous graphs, for the dynamical generation of Majorana masses. The latter scenarios can also be realised in some (compactified) string theory models, where the (totally antisymmetric) torsion is played by the field strength of the spin-one antisymmetric tensor (Kalb-Ramond) field, which exists in the gravitational string multiplet.
\end{abstract}

XVI International Workshop on Neutrino Telescopes,

2-6 March 2015

Palazzo Franchetti âA Istituto Veneto, Venice, Italy

\footnotetext{
* Speaker.

$\dagger$ Currently also at: CERN, Physics Department, Theory Division, Geneva $23 \mathrm{CH}-1211$, Switzerland. This work is supported in part by the London Centre for Terauniverse Studies (LCTS), using funding from the European Research Council via the Advanced Investigator Grant 267352 and by STFC (UK) under the research grant ST/L000326/1.
} 


\section{Introduction and Summary}

The discovery [1] of the Higgs boson at the CERN Large Hadron Collider (LHC) in 2012 constitutes an important milestone for the Ultra-Violet (UV) completion of the Standard Model (SM). Although the so-called Higgs mechanism may well explain the generation of most of the particle masses in the SM, the origin of the small neutrino masses still remains an open issue. In particular, the observed smallness of the light neutrino masses may naturally be explained through the see-saw mechanism [2], which necessitates the Majorana nature of the light (active) neutrinos and postulates the presence of heavy right-handed Majorana partners of mass $M_{R}$. The right-handed Majorana mass $M_{R}$ is usually considered to be much larger than the lepton or quark masses. The origin of $M_{R}$ has been the topic of several extensions of the SM in the literature, within the framework of quantum field theory [2,3] and string theory [4]. However, up to now, there is no experimental evidence for right-handed neutrinos or for any extension of SM, as a matter of fact, although some optimism of discovering supersymmetry in the next round of LHC (operating at $14 \mathrm{TeV}$ energies) exists among particle physicists.

Until therefore such extensions of the SM are discovered, it is legitimate to search for alternative mechanisms for neutrino mass generation, that keep the spectrum of SM intact, except perhaps for the existence of right handed neutrinos that are allowed. Such minimal, non supersymmetric extensions of the Standard Model with three in fact right-handed Majorana neutrinos complementing the three active left-handed neutrinos (termed $v$ MSM), have been proposed [5], in a way consistent with current cosmology. Such models are characterised by relatively light right-handed neutrinos, two of which are almost degenerate, with masses of order $\mathrm{GeV}$, and a much lighter one, almost decoupled, with masses in the $\mathrm{keV}$ range, which may play the role of warm dark matter. The right-handed neutrinos serve the purpose of generating, , through seesaw type mechanisms, the active neutrino mass spectrum, consistent with observed flavour oscillations. However, there are no suggestions for microscopic mechanisms for the generation of the right-handed neutrino mass spectrum in such scenarios.

Motivated by these facts we review in this talk alternative proposals for neutrino mass generation, through the interaction of neutrinos with non-trivial backgrounds. We examine two such types of backgrounds. The first [6], to be discussed in section 2, violates Lorentz symmetry spontaneously. The background is of a type existing in the so-called Lorentz-Violating (LV) Standard Model Extension (SME) of Kostelecky and collaborators [7]; it can be associated (but this is only one example) with some string/brane models of the Universe, in which our four space-time dimensional brane world propagates in a bulk punctured by populations of point-like D-brane defects, interacting with right-handed neutrinos [8]. The second proposal for dynamical generation of neutrino masses concerns their propagation in space-time geometries with quantum-fluctuating torsion [9] and is discussed in section 3. The totally antisymmetric part of the torsion couples, via the gravitational covariant derivative, to all fermions in a way that the resulting interaction resembles that of the Lorentz and CPT-Violating pseudovector background with the axial fermion current in the SME. The generation of (right-handed, sterile) neutrino masses in that case proceeds, as we shall review below, via chiral anomalous three-loop graphs of neutrinos interacting with the totally antisymmetric torsion quantum field. In four space-time dimensions, the latter is represented as an axion field, whose mixing with ordinary axion fields, that in turn interact with the Majorana 
right-handed neutrinos via chirality changing Yukawa couplings, is held responsible for the righthanded Majorana neutrino mass generation through the aforementioned anomalous graphs. This latter scenario may be motivated by some string-inspired proposals [10] for leptogenesis induced by the torsion background in geometries of the early universe, which we do not discuss here, due to lack of space. The totally antisymmetric torsion in such cases corresponds to the field strength of the spin-one antisymmetric tensor (Kalb-Ramond) field of the string gravitational multiplet.

\section{Neutrino Mass Generation due to propagation in Lorentz Violating Backgrounds}

The model to be considered in this part of the talk is defined by the two-flavour fermion Lagrangian

$$
\mathscr{L}_{2}=\bar{\Psi}\left[i\left(\partial_{0} \gamma^{0}-\vec{\partial} \cdot \vec{\gamma}\right)\left(1-\frac{\Delta}{M^{2}}\right)+\frac{\Delta}{M}\right] \Psi+\frac{1}{M^{2}}(\bar{\Psi} \tau \Psi)^{2},
$$

where $\Psi=\left(\begin{array}{l}\psi_{1} \\ \psi_{2}\end{array}\right)$ is a fermion flavour doublet, with bare mass zero, and

$$
\tau=\left(\begin{array}{ll}
g_{1} & g_{3} \\
g_{3} & g_{2}
\end{array}\right)
$$

is the (dimensionless) interaction coupling matrix. The mass scale $M$ is used both to control the LV scale and the strength of the four-fermion interaction ${ }^{1}$. We shall argue that in this model fermion masses and flavour oscillations are generated dynamically.

The model belongs to an SME of the type

$$
\mathscr{L}_{S M E}=\bar{\psi}(i \not \partial-m+Q) \psi,
$$

where $Q$ contains the $\mathrm{LV}$ terms, and can be expanded on a basis of gamma matrices $Q=A+i B \gamma^{5}+$ $C_{\mu} \gamma^{\mu}+D_{\mu} \gamma^{\mu} \gamma^{5}+E_{\mu \nu} \sigma^{\mu \nu}$, with $\sigma^{\mu \nu}=\frac{1}{4}\left[\gamma^{\mu} \cdot \gamma^{\nu}\right]$. The (tensorial) quantities $A, B, C_{\mu}, D_{\mu}, E_{\mu \nu}$ may contain any number of derivatives, including terms which are either odd or even under the discrete symmetry CPT. The different coefficients associated with these terms can arise from vacuum expectation values (vev) of tensors of different ranks, and should satisfy upper bounds for Lorentz symmetry violation, imposed by experiments [7]. The model (2.1) corresponds to the specific case $A=\frac{b}{M} \Delta, C_{0}=-i \frac{a}{M^{2}} \Delta \partial_{0}, \vec{C}=-i \frac{c}{M^{2}} \Delta \vec{\partial}, B=D_{\mu}=E_{\mu \nu}=0$, where $a, b, c$ are dimensionless constants $(a>0$ and $c>0)$, such that $Q=-i \partial_{0} \gamma^{0} \frac{a}{M^{2}} \Delta+i \vec{\partial} \cdot \vec{\gamma}\left(i \frac{b}{M} \vec{\partial} \cdot \vec{\gamma}+\frac{c}{M^{2}} \Delta\right)$. The relevant case for our study corresponds to non-vanishing coefficients $a, b, c$, which leads [6] to a quasi-relativistic dispersion relation, in the sense that it is relativistic in both the infrared and the ultraviolet, but not in an intermediate regime, characterized by the mass scale $M$, and the absence of critical coupling for the generation of dynamical mass.

The choice (2.1) is motivated by a gravitational microscopic model, based on the low-energy limit of a string theory on a three brane universe, which is embedded, from an effective threebrane observer view point, in a bulk space-time punctured with point-like defects (D-particles) [8].

\footnotetext{
${ }^{1}$ When applied to right-handed neutrinos, and in cases, such as the $v$ MSM [5], where the lightest of them (of keV mass) plays the role of dark matter, the four fermion interactions provide an example of self-interacting dark matter, which may play an important rôle in galactic structure [11]
} 
Non-trivial interactions of D-particles with stringy matter occur only for such matter that does not carry standard model quantum numbers, and from this point of view right-handed sterile neutrinos constitute perfect candidates. As shown in [6], this model provides an elegant construction of the LV operator $Q$ from a fundamental theory, which allows the generation of the operator $Q$ in a natural way. In this microscopic context, the Lorentz symmetric limit $M \rightarrow \infty$, which we shall consider in this work and by means of which one views the LV as a catalyst of mass generation, follows when the density of D-particles becomes vanishingly small.

To study dynamical generation of fermion masses, we introduce a Yukawa coupling of fermions to an auxiliary field $\phi$, which is used for the linearisation of the four-fermion interactions in (2.1), integrate over the fermions in a path integral, and look for a non-trivial minimum for the effective potential $V(\phi)$. This leads to a mass term in the original Yukawa interaction. This approach neglects fluctuations of the auxiliary field about its vacuum expectation value (vev), but these can be omitted in the limit $g^{2} \rightarrow 0$, which characterises the Lorentz symmetric limit ${ }^{2}$.

The Lagrangian containing the auxiliary field, equivalent to the original Lagrangian (2.1), reads

$$
\mathscr{L}_{2}^{\prime}=\bar{\Psi}\left[i\left(\partial_{0} \gamma^{0}-\vec{\partial} \cdot \vec{\gamma}\right)\left(1-\frac{\Delta}{M^{2}}\right)+\frac{\Delta}{M}\right] \Psi-\frac{M^{2}}{4} \phi^{2}-\phi \bar{\Psi} \tau \Psi
$$

Integrating over fermions, we obtain the effective potential for the auxiliary field $\phi[6] V_{2}=\frac{M^{2}}{4} \phi^{2}+$ $i \operatorname{tr} \int \frac{d^{4} p}{(2 \pi)^{4}}\left(\ln \lambda_{+}+\ln \lambda_{-}\right)$, where $\lambda_{ \pm}=\left(\omega \gamma^{0}-\vec{p} \cdot \vec{\gamma}\right)\left(1+p^{2} / M^{2}\right)-p^{2} / M-h_{ \pm} \phi, \omega$ is the frequency, $p \equiv|\vec{p}|$, and $h_{ \pm}=\frac{1}{2}\left(g_{1}+g_{2}\right) \pm \frac{1}{2} \sqrt{\left(g_{1}-g_{2}\right)^{2}+4 g_{3}^{2}}$ are the eigenvalues of the coupling matrix.

Minimization $\left.\left(d V_{2} / d \phi\right)\right|_{\phi=\langle\phi\rangle \equiv \phi_{2}}=0$ leads to a gap equation for the fermion masses

$$
\frac{M^{2}}{2} \phi_{2}=\sum_{s=+,-} \frac{h_{s}}{\pi^{3}} \int p^{2} d p \int d \omega\left[\frac{\left(h_{s} \phi_{2}+p^{2} / M\right)}{\left(\omega^{2}+p^{2}\right)\left(1+p^{2} / M^{2}\right)^{2}+\left(h_{s} \phi_{2}+p^{2} / M\right)^{2}}\right]
$$

From (2.5) it follows that the dynamically generated mass matrix $\mathscr{M}=\phi_{2} \tau$ (cf. (2.4)) reads in the (Lorentz-symmetric) limit of small couplings $g_{i} \ll 1$ we are interested in here,

$$
\mathscr{M} \simeq 0.018\left(g_{1}+g_{2}\right) M\left(\begin{array}{ll}
g_{1} & g_{3} \\
g_{3} & g_{2}
\end{array}\right) \text {. }
$$

The mass eigenvalues $m_{ \pm}=\phi_{2} h_{ \pm}$and the mixing angle $\theta$ are then given by

$$
\begin{aligned}
m_{ \pm} & =0.009 M\left[\left(g_{1}+g_{2}\right)^{2} \pm \sqrt{\left(g_{1}^{2}-g_{2}^{2}\right)^{2}+4 g_{3}^{2}\left(g_{1}+g_{2}\right)^{2}}\right] \\
\tan \theta & =\frac{g_{1}-g_{2}}{2 g_{3}}+\sqrt{1+\left(\frac{g_{1}-g_{2}}{2 g_{3}}\right)^{2}} .
\end{aligned}
$$

\footnotetext{
${ }^{2}$ In terms of the microscopic, string-inspired model [8], the coupling is proportional to the density of D-particle defects, and hence its vanishing is consistent with the simultaneous limit $M \rightarrow \infty$, since $M$ is inversely proportional to the defects density. Hence the Lorentz symmetric limit of that model corresponds to vanishing D-particle-defect density.
} 
From this we can express the dimensionless couplings $g_{i}$ as

$$
\begin{aligned}
& g_{1}=\frac{\mu_{+}+\mu_{-}+\left(\mu_{+}-\mu_{-}\right) \cos (2 \theta)}{2 \sqrt{\alpha\left(\mu_{+}+\mu_{-}\right)}}, \\
& g_{2}=\frac{\mu_{+}+\mu_{-}-\left(\mu_{+}-\mu_{-}\right) \cos (2 \theta)}{2 \sqrt{\alpha\left(\mu_{+}+\mu_{-}\right)}}, \\
& g_{3}=\frac{\mu_{-}-\mu_{+}}{2 \sqrt{\alpha\left(\mu_{+}+\mu_{-}\right)}} \sin (2 \theta),
\end{aligned}
$$

where $\mu_{ \pm}=\frac{m_{ \pm}}{M}$. Therefore one can write the couplings $g_{i}$ in the form

$$
g_{i}=\frac{a_{i}}{\sqrt{M}}, i=1,2,3,
$$

where the constants $a_{i}$ are kept finite and are fixed by the "experimental" (in case of realistic models) values of $m_{ \pm}$and $\theta$. This expression shows the explicit dependence of the couplings $g_{i}$ on the scale $M$, for the Lorentz symmetric limit $M \rightarrow \infty$ to be taken, in such a way that we are left with two relativistic free fermions, for which flavour oscillations have been generated dynamically. Therefore any set of values for $m_{ \pm}$and $\theta$ can be described by the Lorentz-symmetric limit of our model, by considering the coupling constants (2.8).

The model can be straightforwardly extended to Majorana fermions, as well as three generations of fermions, including seesaw type Lagrangians, where by a judicious choice of the appropriate couplings one can generate, in the Lorentz-symmetric limit, (right-handed) neutrino mass hierarchies of relevance, e.g. to $v \mathrm{MSM}$ and warm dark matter studies [5].

\section{Neutrino Mass Generation due to Interactions with Quantum Torsion}

Let us for concreteness consider Dirac fermions in a torsionful space-time. The extension to the Majorana case is straightforward. The relevant action reads:

$$
S_{\psi}=\frac{i}{2} \int d^{4} x \sqrt{-g}\left(\bar{\psi} \gamma^{\mu} \overline{\mathscr{D}}_{\mu} \psi-\left(\overline{\mathscr{D}}_{\mu} \bar{\psi}\right) \gamma^{\mu} \psi\right)
$$

where $\overline{\mathscr{D}}_{\mu}=\bar{\nabla}_{\mu}+\ldots$, is the covariant derivative (including gravitational and gauge-field connection parts, in case the fermions are charged). The overline above the covariant derivative, i.e. $\bar{\nabla}_{\mu}$, denotes the presence of torsion, which is introduced through the torsionful spin connection: $\bar{\omega}_{a b \mu}=\omega_{a b \mu}+K_{a b \mu}$, where $K_{a b \mu}$ is the contorsion tensor. The latter is related to the torsion twoform $\mathbf{T}^{a}=\mathbf{d} \mathbf{e}^{a}+\bar{\omega}^{a} \wedge \mathbf{e}^{b}$ via [12,13]: $K_{a b c}=\frac{1}{2}\left(\mathrm{~T}_{c a b}-\mathrm{T}_{a b c}-\mathrm{T}_{b c d}\right)$. The presence of torsion in the covariant derivative in the action (3.1) leads, apart from the standard terms in manifolds without torsion, to an additional term involving the axial current

$$
J_{5}^{\mu} \equiv \bar{\psi} \gamma^{\mu} \gamma^{5} \psi
$$

The relevant part of the action reads:

$$
S_{\psi} \ni-\frac{3}{4} \int d^{4} \sqrt{-g} S_{\mu} \bar{\psi} \gamma^{\mu} \gamma^{5} \psi=-\frac{3}{4} \int S \wedge^{\star} J^{5}
$$


where $\mathbf{S}={ }^{\star} \mathbf{T}$ is the dual of $\mathbf{T}: S_{d}=\frac{1}{3 !} \varepsilon^{a b c}{ }_{d} T_{a b c}$.

We next remark that the torsion tensor can be decomposed into its irreducible parts [12], of which $S_{d}$ is the pseudoscalar axial vector: $T_{\mu v \rho}=\frac{1}{3}\left(T_{v} g_{\mu \rho}-T_{\rho} g_{\mu \nu}\right)-\frac{1}{3 !} \varepsilon_{\mu \nu \rho \sigma} S^{\sigma}+q_{\mu \nu \rho}$, with $\varepsilon_{\mu \nu \rho \sigma} q^{v \rho \sigma}=q_{\rho v}^{v}=0$. This implies that the contorsion tensor undergoes the following decomposition:

$$
K_{a b c}=\frac{1}{2} \varepsilon_{a b c d} S^{d}+\widehat{K}_{a b c}
$$

where $\widehat{K}$ includes the trace vector $T_{\mu}$ and the tensor $q_{\mu v \rho}$ parts of the torsion tensor.

The gravitational part of the action can then be written as: $S_{G}=\frac{1}{2 \kappa^{2}} \int d^{4} x \sqrt{-g}(R+\widehat{\Delta})+$ $\frac{3}{4 \kappa^{2}} \int \mathbf{S} \wedge{ }^{\star} \mathbf{S}$, where $\widehat{\Delta}=\widehat{K}_{\mu \nu}^{\lambda} \widehat{K}_{\lambda}^{\nu \mu}-\widehat{K}^{\mu \nu}{ }_{\nu} \widehat{K}_{\mu \lambda}{ }^{\lambda}$, with the hatted notation defined in (3.4).

In a quantum gravity setting, where one integrates over all fields, the torsion terms appear as non propagating fields and thus they can be integrated out exactly. The authors of [13] have observed though that the classical equations of motion identify the axial-pseudovector torsion field $S_{\mu}$ with the axial current, since the torsion equation yields

$$
K_{\mu a b}=-\frac{1}{4} e_{\mu}^{c} \varepsilon_{a b c d} \bar{\psi} \gamma_{5} \tilde{\gamma}^{d} \psi
$$

From this it follows $\mathbf{d}^{\star} \mathbf{S}=0$, leading to a conserved "torsion charge" $Q=\int{ }^{\star} \mathbf{S}$. To maintain this conservation in quantum theory, they postulated $\mathbf{d}^{\star} \mathbf{S}=0$ at the quantum level, which can be achieved by the addition of judicious counter terms. This constraint, in a path-integral formulation of quantum gravity, is then implemented via a delta function constraint, $\delta\left(d^{\star} \mathbf{S}\right)$, and the latter via the well-known trick of introducing a Lagrange multiplier field $\Phi(x) \equiv\left(3 / \kappa^{2}\right)^{1 / 2} b(x)$. Hence, the relevant torsion part of the quantum-gravity path integral would include a factor

$$
\begin{aligned}
& \mathscr{Z} \propto \int D \mathbf{S} D b \exp \left[i \int \frac{3}{4 \kappa^{2}} \mathbf{S} \wedge^{\star} \mathbf{S}-\frac{3}{4} \mathbf{S} \wedge^{\star} \mathbf{J}^{5}+\left(\frac{3}{2 \kappa^{2}}\right)^{1 / 2} b d^{\star} \mathbf{S}\right] \\
& =\int D b \exp \left[-i \int \frac{1}{2} \mathbf{d} b \wedge^{\star} \mathbf{d} b+\frac{1}{f_{b}} \mathbf{d} b \wedge^{\star} \mathbf{J}^{5}+\frac{1}{2 f_{b}^{2}} \mathbf{J}^{5} \wedge^{\star} \mathbf{J}^{5}\right],
\end{aligned}
$$

where $f_{b}=\left(3 \kappa^{2} / 8\right)^{-1 / 2}=\frac{M_{P}}{\sqrt{3 \pi}}$ and the non-propagating $\mathbf{S}$ field has been integrated out. The reader should notice that, as a result of this integration, the corresponding effective field theory contains a non-renormalizable repulsive four-fermion axial current-current interaction, characteristic of any torsionful theory [12].

The torsion term, being geometrical, due to gravity, couples universally to all fermion species, not only neutrinos. Thus, in the context of the SM of particle physics, the axial current (3.2) is expressed as a sum over fermion species

$$
J_{5}^{\mu} \equiv \sum_{i=\text { fermion species }} \bar{\psi}_{i} \gamma^{\mu} \gamma^{5} \psi_{i}
$$

In theories with chiral anomalies, like the quantum electrodynamics part of SM, the axial current is not conserved at the quantum level, due to anomalies, but its divergence is obtained by the one-loop result [14]:

$$
\begin{aligned}
\nabla_{\mu} J^{5 \mu} & =\frac{e^{2}}{8 \pi^{2}} F^{\mu v} \widetilde{F}_{\mu v}-\frac{1}{192 \pi^{2}} R^{\mu v \rho \sigma} \widetilde{R}_{\mu v \rho \sigma} \\
& \equiv G(\mathbf{A}, \omega) .
\end{aligned}
$$


We may then partially integrate the second term in the exponent on the right-hand-side of (3.6) and take into account (3.8). The reader should observe that in (3.8) the torsion-free spin connection has been used. This can be achieved by the addition of proper counter terms in the action [13], which can convert the anomaly from the initial $G(\mathbf{A}, \bar{\omega})$ to $G(\mathbf{A}, \omega)$. Using (3.8) in (3.6) one can then obtain for the effective torsion action in theories with chiral anomalies, such as the QED part of the SM:

$$
\int D b \exp \left[-i \int \frac{1}{2} \mathbf{d} b \wedge^{\star} \mathbf{d} b-\frac{1}{f_{b}} b G(\mathbf{A}, \omega)+\frac{1}{2 f_{b}^{2}} \mathbf{J}^{5} \wedge^{\star} \mathbf{J}^{5}\right]
$$

A concrete example of torsion is provided by string-inspired theories, where the totally antisymmetric component $S_{\mu}$ of the torsion is identified with the field strength of the spin-one antisymmetric tensor (Kalb-Ramond (KR) [15]) field $H_{\mu v \rho}=\partial_{[\mu} B_{v \rho]}$, where the symbol [...] denotes antisymmetrization of the appropriate indices. The string theory effective action depends only on $H_{\mu v \rho}$ as a consequence of the "gauge symmetry" $B_{\mu \nu} \rightarrow B_{\mu \nu}+\partial_{[\mu} \Theta_{v]}$ that characterises all string theories. It can be shown [16] that the terms of the effective action up to and including quadratic order in the Regge slope parameter $\alpha^{\prime}$, of relevance to the low-energy (field-theory) limit of string theory, which involve the $\mathrm{H}$-field strength, can be assembled in such a way that only torsionful Christoffel symbols, $\bar{\Gamma}_{v \rho}^{\mu}$ appear: $\bar{\Gamma}_{v \rho}^{\mu}=\Gamma_{v \rho}^{\mu}+\frac{\kappa}{\sqrt{3}} H_{v \rho}^{\mu} \neq \bar{\Gamma}_{\rho v}^{\mu}$, where $\Gamma_{v \rho}^{\mu}=\Gamma_{\rho v}^{\mu}$ is the ordinary, torsion-free, symmetric connection, and $\kappa$ is the gravitational constant. In four space-time dimensions, the dual of the $\mathrm{H}$-field is indeed the derivative of an axion-like field, analogous to the field $b$ above. For completeness we mention at this point that background geometries with (approximately) constant background $H_{i j k}$ torsion, where Latin indices denote spatial components of the four-dimensional space-time, may characterise the early universe. In such cases, the H-torsion background constitutes extra source of $\mathrm{CP}$ violation, necessary for lepotogenesis, and through Baryon-minus-Lepton-number (B-L) conserving processes, Baryogenesis, and thus the observed matter-antimatter asymmetry in the Universe [10]. Today of course any torsion background should be strongly suppressed, due to the lack of any experimental evidence for it. Scenarios as to how such cosmologies can evolve so as to guarantee the absence of any appreciable traces of torsion today can be found in [10].

In what follows we shall consider the effects of the quantum fluctuations of torsion, which survive the absence of any torsion background. An important aspect of the coupling of the torsion (or KR axion) quantum field $b(x)$ to the fermionic matter discussed above is its shift symmetry, characteristic of an axion field. Indeed, by shifting the field $b(x)$ by a constant: $b(x) \rightarrow b(x)+c$, the action (3.9) only changes by total derivative terms, such as $c R^{\mu \nu \rho \sigma} \widetilde{R}_{\mu \nu \rho \sigma}$ and $c F^{\mu \nu} \widetilde{F}_{\mu \nu}$. These terms are irrelevant for the equations of motion and the induced quantum dynamics, provided the fields fall off sufficiently fast to zero at space-time infinity. The scenario for the anomalous Majorana mass generation through torsion proposed in [9], and reviewed here, consists of augmenting the effective action (3.9) by terms that break such a shift symmetry. To illustrate this last point, we first couple the KR axion $b(x)$ to another pseudoscalar axion field $a(x)$. In string-inspired models, such pseudoscalar axion $a(x)$ may be provided by the string moduli [17]. The proposed coupling occurs through a mixing in the kinetic terms of the two fields. To be specific, we consider the action 
(henceforth we restrict ourselves to right-handed Majorana neutrino fermion fields)

$$
\begin{aligned}
\mathscr{S}= & \int d^{4} x \sqrt{-g}\left[\frac{1}{2}\left(\partial_{\mu} b\right)^{2}+\frac{b(x)}{192 \pi^{2} f_{b}} R^{\mu v \rho \sigma} \widetilde{R}_{\mu v \rho \sigma}+\frac{1}{2 f_{b}^{2}} J_{\mu}^{5} J^{5^{\mu}}+\gamma\left(\partial_{\mu} b\right)\left(\partial^{\mu} a\right)+\frac{1}{2}\left(\partial_{\mu} a\right)^{2}\right. \\
& \left.-y_{a} i a\left(\bar{\psi}_{R}^{C} \psi_{R}-\bar{\psi}_{R} \psi_{R}^{C}\right)\right],
\end{aligned}
$$

where $\psi_{R}^{C}=\left(\psi_{R}\right)^{C}$ is the charge-conjugate right-handed fermion $\psi_{R}, J_{\mu}^{5}=\bar{\psi} \gamma_{\mu} \gamma_{5} \psi$ is the axial current of the four-component Majorana fermion $\psi=\psi_{R}+\left(\psi_{R}\right)^{C}$, and $\gamma$ is a real parameter to be constrained later on. Here, we have ignored gauge fields, which are not of interest to us, and the possibility of a non-perturbative mass $M_{a}$ for the pseudoscalar field $a(x)$. Moreover, we remind the reader that the repulsive self-interaction fermion terms are due to the existence of torsion in the Einstein-Cartan theory. The Yukawa coupling $y_{a}$ of the axion moduli field $a$ to right-handed sterile neutrino matter $\psi_{R}$ may be due to non perturbative effects. These terms break the shift symmetry: $a \rightarrow a+c$.

It is convenient to diagonalize the axion kinetic terms by redefining the KR axion field as follows: $b(x) \rightarrow b^{\prime}(x) \equiv b(x)+\gamma a(x)$. This implies that the effective action (3.10) becomes:

$$
\begin{aligned}
& \mathscr{S}=\int d^{4} x \sqrt{-g}\left[\frac{1}{2}\left(\partial_{\mu} b^{\prime}\right)^{2}+\frac{1}{2}\left(1-\gamma^{2}\right)\left(\partial_{\mu} a\right)^{2}\right. \\
& \left.+\frac{1}{2 f_{b}^{2}} J_{\mu}^{5} J^{5^{\mu}}+\frac{b^{\prime}(x)-\gamma a(x)}{192 \pi^{2} f_{b}} R^{\mu v \rho \sigma} \widetilde{R}_{\mu v \rho \sigma}-y_{a} i a\left(\bar{\psi}_{R}^{C} \psi_{R}-\bar{\psi}_{R} \psi_{R}^{C}\right)\right] .
\end{aligned}
$$

Thus we observe that the $b^{\prime}$ field has decoupled and can be integrated out in the path integral, leaving behind an axion field $a(x)$ coupled both to matter fermions and to the operator $R^{\mu v \rho \sigma} \widetilde{R}_{\mu v \rho \sigma}$, thereby playing now the rôle of the torsion field. We observe though that the approach is only valid for $|\gamma|<1$, otherwise the axion field would appear as a ghost, i.e. with the wrong sign of its kinetic terms, which would indicate an instability of the model. This is the only restriction of the parameter $\gamma$. In this case we may redefine the axion field so as to appear with a canonical normalised kinetic term, implying the effective action:

$$
\begin{gathered}
\mathscr{S}_{a}=\int d^{4} x \sqrt{-g}\left[\frac{1}{2}\left(\partial_{\mu} a\right)^{2}-\frac{\gamma a(x)}{192 \pi^{2} f_{b} \sqrt{1-\gamma^{2}}} R^{\mu v \rho \sigma} \widetilde{R}_{\mu v \rho \sigma}\right. \\
\left.-\frac{i y_{a}}{\sqrt{1-\gamma^{2}}} a\left(\bar{\psi}_{R}^{C} \psi_{R}-\bar{\psi}_{R} \psi_{R}^{C}\right)+\frac{1}{2 f_{b}^{2}} J_{\mu}^{5} J^{5^{\mu}}\right] .
\end{gathered}
$$

Evidently, the action $\mathscr{S}_{a}$ in (3.12) corresponds to a canonically normalised axion field $a(x)$, coupled both to the curvature of space-time, à la torsion, with a modified coupling $\gamma /\left(192 \pi^{2} f_{b} \sqrt{1-\gamma^{2}}\right)$, and to fermionic matter with chirality-changing Yukawa-like couplings of the form $y_{a} / \sqrt{1-\gamma^{2}}$.

The mechanism for the anomalous Majorana mass generation is shown in Fig. 1. We may now estimate the two-loop Majorana neutrino mass in quantum gravity with an effective UV energy cutoff $\Lambda$. Adopting the effective field-theory framework of [18], the gravitationally induced Majorana mass for right-handed neutrinos, $M_{R}$, is estimated to be:

$$
M_{R} \sim \frac{1}{\left(16 \pi^{2}\right)^{2}} \frac{y_{a} \gamma \kappa^{4} \Lambda^{6}}{192 \pi^{2} f_{b}\left(1-\gamma^{2}\right)}=\frac{\sqrt{3} y_{a} \gamma \kappa^{5} \Lambda^{6}}{49152 \sqrt{8} \pi^{4}\left(1-\gamma^{2}\right)} .
$$

In a UV complete theory such as strings, the cutoff $\Lambda$ and the Planck mass scale $M_{P}$ are related. 


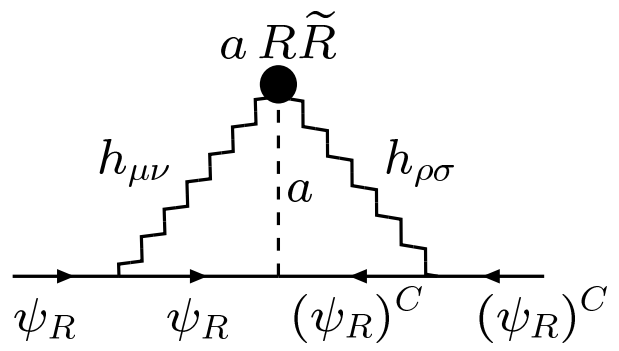

Figure 1: Typical Feynman graph giving rise to anomalous fermion mass generation [9]. The black circle denotes the operator $a(x) R_{\mu \nu \lambda \rho} \widetilde{R}^{\mu \nu \lambda \rho}$ induced by torsion. The fields $h_{\mu v}$ denote graviton fluctuations.

It is interesting to provide a numerical estimate of the anomalously generated Majorana mass $M_{R}$. Assuming that $\gamma \ll 1$, the size of $M_{R}$ may be estimated from (3.13) to be

$$
M_{R} \sim\left(3.1 \times 10^{11} \mathrm{GeV}\right)\left(\frac{y_{a}}{10^{-3}}\right)\left(\frac{\gamma}{10^{-1}}\right)\left(\frac{\Lambda}{2.4 \times 10^{18} \mathrm{GeV}}\right)^{6} .
$$

Obviously, the generation of $M_{R}$ is highly model dependent. Taking, for example, the quantum gravity scale to be $\Lambda=10^{17} \mathrm{GeV}$, we find that $M_{R}$ is at the TeV scale, for $y_{a}=10^{-3}$ and $\gamma=0.1$. However, if we take the quantum gravity scale to be close to the GUT scale, i.e. $\Lambda=10^{16} \mathrm{GeV}$, we obtain a right-handed neutrino mass $M_{R} \sim 16 \mathrm{keV}$, for the choice $y_{a}=\gamma=10^{-3}$. This is in the preferred ballpark region for the sterile neutrino $\psi_{R}$ to qualify as a warm dark matter [19].

In a string-theoretic framework, many axions might exist that could mix with each other [17]. Such a mixing can give rise to reduced UV sensitivity of the two-loop graph shown in Fig. 1. In such cases, the anomalously generated Majorana mass may be estimated to be:

$M_{R} \sim \frac{\sqrt{3} y_{a} \gamma \kappa^{5} \Lambda^{6-2 n}\left(\delta M_{a}^{2}\right)^{n}}{49152 \sqrt{8} \pi^{4}\left(1-\gamma^{2}\right)}$ for $n \leq 3$, and $M_{R} \sim \frac{\sqrt{3} y_{a} \gamma \kappa^{5}\left(\delta M_{a}^{2}\right)^{3}}{49152 \sqrt{8} \pi^{4}\left(1-\gamma^{2}\right)} \frac{\left(\delta M_{a}^{2}\right)^{n-3}}{\left(M_{a}^{2}\right)^{n-3}}$ for $n>3$.

It is then not difficult to see that three axions $a_{1,2,3}$ with next-to-neighbour mixing as discussed above would be sufficient to obtain a UV finite result for $M_{R}$ at the two-loop level. Of course, beyond the two loops, $M_{R}$ will depend on higher powers of the energy cut-off $\Lambda$, i.e. $\Lambda^{n>6}$, but if $\kappa \Lambda \ll 1$, these higher-order effects are expected to be subdominant.

In the above $n$-axion-mixing scenarios, we note that the anomalously generated Majorana mass term will only depend on the mass-mixing parameters $\delta M_{a}^{2}$ of the axion fields and not on their masses themselves, as long as $n \leq 3$. As a final comment we mention that the values of the Yukawa couplings $y_{a}$ may be determined by some underlying discrete symmetry [20], which for instance allows two of the right-handed neutrinos to be almost degenerate in mass, as required for enhanced CP violation of relevance to leptogenesis [10], or in general characterises the vMSM [5]. These are interesting issues that deserve further exploration.

\section{References}

[1] G. Aad et al. [ATLAS Collaboration], Phys. Lett. B 716, 1 (2012) [arXiv:1207.7214 [hep-ex]]; S. Chatrchyan et al. [CMS Collaboration], Phys. Lett. B 716, 30 (2012) [arXiv:1207.7235 [hep-ex]].

[2] P. Minkowski, Phys. Lett. B 67, 421 (1977); M. Gell-Mann, P. Ramond and R. Slansky, in Supergravity, eds. D.Z. Freedman and P. van Nieuwenhuizen (North-Holland, Amsterdam, 1979); T. Yanagida, in Proc. of the Workshop on the Unified Theory and the Baryon Number in the Universe, 
Tsukuba, Japan, 1979, eds. O. Sawada and A. Sugamoto; R. N. Mohapatra and G. Senjanović, Phys. Rev. Lett. 44, 912 (1980).

[3] J. Schechter and J. W. F. Valle, Phys. Rev. D 22, 2227 (1980); G. Lazarides, Q. Shafi and C. Wetterich, Nucl. Phys. B 181, 287 (1981); For recent reviews see: R. N. Mohapatra et al., "Theory of Neutrinos: a White Paper,” Rept. Prog. Phys. 70, 1757 (2007) [hep-ph/0510213] and references therein.

[4] R. Blumenhagen, M. Cvetic and T. Weigand, Nucl. Phys. B 771, 113 (2007) [hep-th/0609191]; M. Cvetic, R. Richter and T. Weigand, Phys. Rev. D 76, 086002 (2007) [hep-th/0703028].

[5] T. Asaka, M. Shaposhnikov and A. Kusenko, “Opening a New Window for Warm Dark Matter," Phys. Lett. B 638, 401 (2006); M. Shaposhnikov, JHEP 0808, 008 (2008) [arXiv:0804.4542 [hep-ph]] and references therein.

[6] J. Alexandre, J. Leite and N. E. Mavromatos, J. Alexandre, J. Leite and N. E. Mavromatos, Phys. Rev. D 90, no. 4, 045026 (2014) [arXiv:1404.7429 [hep-th]]; ibid. . D 87, no. 12, 125029 (2013) [arXiv:1304.7706 [hep-ph]] and references therein.

[7] D. Colladay and V. A. Kostelecky, Phys. Rev. D 58, 116002 (1998) [hep-ph/9809521]; V. A. Kostelecky and N. Russell, Rev. Mod. Phys. 83, 11 (2011) [arXiv:0801.0287 [hep-ph]].

[8] N. E. Mavromatos, Phys. Rev. D 83, 025018 (2011) [arXiv:1011.3528 [hep-ph]].

[9] N. E. Mavromatos and A. Pilaftsis, Phys. Rev. D 86, 124038 (2012) [arXiv:1209.6387 [hep-ph]].

[10] N. E. Mavromatos and S. Sarkar, Eur. Phys. J. C 73, no. 3, 2359 (2013) [arXiv:1211.0968 [hep-ph]]. J. Ellis, N. E. Mavromatos and S. Sarkar, Phys. Lett. B 725, 407 (2013) [arXiv:1304.5433 [gr-qc]]. M. de Cesare, N. E. Mavromatos and S. Sarkar, arXiv:1412.7077 [hep-ph].

[11] C. R. Argüelles, N. E. Mavromatos, J. A. Rueda and R. Ruffini, arXiv:1502.00136 [astro-ph.GA].

[12] F. W. Hehl, P. Von Der Heyde, G. D. Kerlick and J. M. Nester, "General Relativity with Spin and Torsion: Foundations and Prospects," Rev. Mod. Phys. 48, 393 (1976);

I. L. Shapiro, "Physical Aspects of the Space-Time Torsion,” Phys. Rept. 357, 113 (2002) [hep-th/0103093] and references therein

[13] M. J. Duncan, N. Kaloper and K. A. Olive, Nucl. Phys. B 387, 215 (1992).

[14] R. Delbourgo and A. Salam, “The Gravitational Correction to PCAC," Phys. Lett. B 40, 381 (1972); see, also: S. Weinberg, The Quantum Theory of Fields. Volume II: Modern Applications. (Cambridge University Press 2001) ISBN 0-521-55002-5.

[15] M. Kalb and P. Ramond, Phys. Rev. D 9, 2273 (1974).

[16] R. R. Metsaev and A. A. Tseytlin, Nucl. Phys. B 293, 385 (1987);

D. J. Gross and J. H. Sloan, Nucl. Phys. B 291, 41 (1987).

[17] A. Arvanitaki, S. Dimopoulos, S. Dubovsky, N. Kaloper and J. March-Russell, Phys. Rev. D 81, 123530 (2010) [arXiv:0905.4720 [hep-th]]; M. Cicoli, M. Goodsell and A. Ringwald, arXiv:1206.0819 [hep-th].

[18] J. F. Donoghue, "General Relativity as an Effective field theory: the Leading Quantum Corrections," Phys. Rev. D 50, 3874 (1994) [gr-qc/9405057].

[19] T. Asaka, M. Shaposhnikov and A. Kusenko, "Opening a New Window for Warm Dark Matter," Phys. Lett. B 638, 401 (2006).

[20] See for instance: G. K. Leontaris and N. D. Vlachos, Mod. Phys. Lett. A 29, 0179 (2014) [arXiv:1309.5785 [hep-ph]], but with their results applied to the right-handed neutrino sector, and references therein. 